\title{
Importancia de Matemática Financiera para los estudiantes de las carreras Económicas y Administrativas
}

\section{Importance of Financial Mathematics for students of Economic and Administrative careers}

\author{
Orlando Zeledón Zeledón ${ }^{1}$
}

Recibido: 03 de marzo de 2018, Aceptado: 25 de octubre 2018

\section{RESUMEN}

Mediante la elaboración de este ensayo se aprecia la importancia que tiene la asignatura de Matemática Financiera para los estudiantes que cursan las diferentes carreras del Departamento de Ciencias Económicas y Administrativas de la UNAN-Managua, FAREM- Estelí. La asignatura en mención, permite al estudiantado adquirir mayores conocimientos y aprendizajes, así como el dominio de competencias académicas que forman parte de las funciones sustantivas en la formación superior que exige esta Universidad para el futuro profesional. El análisis de los contenidos de esta disciplina, constituye para los alumnos, comprender qué son las operaciones financieras, en el sentido de plantear y ejecutar cálculos numéricos, así como la resolución de ejercicios y casos orientados al mercado financiero, además, la toma de decisiones financieras, económicas y administrativas. De igual manera, los contenidos que se desarrollan sirven de base para fortalecer otras asignaturas para alcanzar mejores aprendizajes, así como la creación de competencias una vez que el egresado esté ejerciendo su profesión en el campo laboral.

Palabras claves: Matemáticas Financieras; aprendizaje; desempeño laboral.

\section{ABSTRACT}

This essay highlights the importance of Financial Mathematics as a subject in the curriculum for students of the Department of Economic and Administrative Sciences at UNAN-Managua, FAREM-Estelí. The mentioned above subject allows students to gain more knowledge as well as the mastery of the academic competences required from graduating students of this University. By analyzing the contents of this subject students will understand the different financial operations useful to pose and solve numerical calculations, exercises and cases proper of the financial market, tools useful for financial, economic and administrative decision making. Likewise, the contents of study serve as the basis for strengthening other areas of study to achieve a better learning and the development of competences once the graduate students starts a professional carreer.

Keywords: Financial Mathematics; learning; work performance.

1 Docente del Departamento de Ciencias Económicas y Administrativas, UNAN-Managua/FAREM-Estelí. Correo electrónico: orlanzelz@ gmail.com

Copyright (c) 2018 Revista Multi-Ensayos. 


\section{INTRODUCCIÓN}

El Modelo Educativo de la UNAN- Managua (2011), establece los procesos curriculares de las asignaturas en las diferentes carreras que ofrece esta casa de estudio, las cuales han fortalecido los conocimientos, habilidades y destrezas adquiridas por los estudiantes, así como el desarrollo de actitudes y valores. Las asignaturas contenidas en los planes de estudio de las carrereas tienen como objetivo vincular interdisciplinariamente al estudiante con la realidad que se vive en el contexto laboral.

El objetivo de este ensayo es destacar que la asignatura de Matemática Financiera es de gran utilidad en las diversas carreras como las administrativas, constatables, económicas, de mercadeo y de finanzas que se ofrecen en el Departamento de Ciencias Económicas y Administrativas. La materia es de mucha utilidad en el área académica para los estudiantes, ya que permite en resolver ejercicios, problemas y casos prácticos orientados en el mercado financiero, la cual la podrán vincular con otras asignaturas en años posteriores de su carrera.

\section{DESARROLLO}

Delfin, (2008), define las Matemáticas Financieras como una rama de la Matemática Aplicada que estudia el valor del dinero en el tiempo, al combinar elementos fundamentales como capitales, tasa, tiempo etc., para conseguir un rendimiento o interés, al brindarle herramientas y métodos que permiten tomar la decisión más correcta en el momento de realizar una inversión.

Otra definición, no menos importante que la anterior, refleja un conjunto de técnicas y procedimientos de carácter cuantitativo que sirven para calcular la equivalencia del valor del dinero en cualquier momento en el tiempo. La medición del valor del dinero ayuda a tomar decisiones financieras, es decir; para valorar el premio de prescindir por cierto tiempo, a cierta tasa de interés, de un determinado recurso financiero o capital. (Reyes, Introducción a la Gestión Financiera, 2004)

Es meritorio destacar que las Matemáticas Financieras actualmente tienen varias connotaciones, según la postura del autor, pero todas sus definiciones presentan el mismo fondo u objetivo que va reflejado de acuerdo al fin que se persigue como es el valor del dinero en el tiempo. Como se sabe, el dinero es un activo monetario imprescindible para tomar decisiones en las actividades económicas, ya que representa un medio de pago porque tiene valor para realizar intercambios en compra y venta de bienes y servicios.

Como bien lo expresan los autores antes mencionados, lo que se busca es el valor del dinero en el tiempo, porque no es lo mismo cierta cantidad de dinero que se invierta o se ahorre hoy a lo que se tendrá el día de mañana, ya que el valor del dinero en el tiempo es sin duda el más importante en las Matemáticas Financieras. Esto significa que su uso debe de buscar cierta rentabilidad, porque a través del tiempo el dinero pierde valor adquisitivo por el fenómeno de la inflación y el proceso de devaluación que sucede con nuestra moneda.

Para explicar este punto, se sabe que con frecuencia una persona o empresa siempre se va a enfrentar a diversos problemas con el uso del dinero, se pregunta qué hacer con cierta cantidad de dinero el día de hoy, 
qué decisión deberá tomar con ese dinero, qué alternativa deberá escoger al momento de invertir. Como sabemos, el sujeto o la empresa se encuentran en una incertidumbre al momento de tomar una decisión, por lo que siempre se estará buscando obtener una ganancia o rentabilidad por el dinero que desea invertir. Como lo expresa (Portus, 2005), otro componente básico de las Matemáticas Financieras es el interés o pago de interés que representa, en otras palabras la renta, compensación o réditos que hay pagar por el uso del dinero prestado. También se puede decir que, el interés es el rendimiento que se tiene al invertir en forma productiva el dinero; ello implica que el interés es la tasa o rentabilidad que se obtiene al invertir un capital.

Sobre estos dos componentes antes descritos, se centra el estudio de las Matemáticas Financieras y de ahí su importancia de cursarla como asignatura en las carreras que ofrece la UNAN Managua, caso particular en la FAREM Estelí, ya que forma parte de su estudio y es un requisito dentro del pénsum de materias de la formación universitaria de los futuros profesionales de las diversas carreras que ofrece el Departamento de Ciencias Económicas y Administrativas entre las cuales figuran: Administración de Empresas, Mercadotecnia, Contaduría Pública y Finanzas, Banca y Finanzas, y Economía.

Sumado a los antes mencionados, se requiere que los estudiantes dominen de las Matemáticas Financieras, otros conceptos básicos, tales como: crédito, ahorro, inversiones, descuentos, depreciación, utilización de documentos financieros; entre estos destacan: pagaré, letra de cambio, bonos, pago de cupones, acciones, certificados de inversiones, análisis de préstamos en diferentes condiciones tasas de interés nominales y efectivas a corto y largo plazo, elaboración de tablas de amortización aplicados a los créditos, análisis de inversiones, operaciones de seguros, análisis financieros y contables; en suma, herramientas fundamentales en las actividades de la vida profesional de un administrador, economista, financista, contador, o profesiones afines.

Otro aspecto que conviene destacar, sobre la importancia de esta asignatura para los estudiantes de estas carreras, es la definición planteada por (López, 2012), quien concibe que las Matemáticas Financieras son importantes para la formación profesional de cualquier individuo que realiza actividades comerciales o bien financieras.

Lo expresado por el autor se concreta en preparar al estudiante con los conocimientos teóricos-prácticos necesarios para el planteamiento, la solución y el análisis de las distintas operaciones financieras que actualmente existen en el mercado, así como sentar las bases para adquirir mejores competencias para desarrollar su actividad profesional en ámbitos laborales; ya sea ejercer su carrera como agente o asesor financiero, en departamentos comerciales y técnicos de entidades bancarias o cooperativas financieras, agentes financieros de entidades públicas y privadas, responsable del área de crédito y financiación de organismos(ONG), entre otras ocupaciones.

Por tanto, es posible que algunos estudiantes, al tener los conocimientos claros de esta de esta asignatura, obtengan su título universitario de la carrera que cursan, y deseen emprender su propio negocio, o si ya lo tienen, pueden poner en práctica los conocimientos adquiridos y los aprendizajes orientados en las diferentes temáticas desarrolladas sobre los contenidos de esta materia cursada, que le ofrece buenas oportunidades de crecer y competir en el mercado. 
De esta manera, como Facultad estamos otorgando al estudiantado una formación básica, que aporta destrezas y habilidades necesarias para el estudio y la resolución de problemas y el uso de todas aquellas operaciones que se realizan en los mercados bursátiles y financieros, aplicables e imprescindibles para otras disciplinas como la contabilidad. Así, se aporta información sobre las operaciones comerciales realizadas por la empresa, lo que y permite la toma de decisiones acertadas al momento de realizar una inversión, la cual se refleja en los estados financieros.

Para la economía, con el uso adecuado de la Matemáticas Financieras, se aporta valiosa información sobre la aplicación de los tipos de interés que se presentan en las apreciaciones y depreciaciones de nuestra moneda con una divisa, así como tomar decisiones relacionadas al fenómeno de la inflación y la devaluación, que afectan los tipos de cambio en las exportaciones e importaciones de bienes y servicios y el costo de poder adquisitivo.

En tanto, para la mercadotecnia, dicha asignatura aporta información relevante para toma de decisiones relacionada a la apertura de nuevos mercados, en cuanto a las ventas y la publicidad, las cuales se ven enormemente beneficiadas, pues una gran parte de los anuncios que se muestran en los medios publicitarios hacen clara referencias a promociones y descuentos, tomando como indicador las atractivas tasas de interés. Para quienes estudian Administración de Empresas, los conocimientos adquiridos en Matemática Financiera, les proporcionan nuevas técnicas de planificación y control, así como el empleo de los recursos materiales, financieros y humanos; además de las regulaciones a las compensaciones salariales relacionadas al departamento del capital humano de quienes laboran en una organización.

En el área de Banca y Finanzas es muy útil para tomar decisiones relacionadas a los préstamos y los financiamientos, tomando en cuenta los plazos, tipos de interés y tasa de interés, así como el análisis de las inversiones, tomando en cuenta su rentabilidad.

En suma, es importante el manejo eficiente de las Matemáticas Financieras, ya que la consolidación en sus conceptos esenciales, brinda al futuro profesional de esta Facultad, las habilidades necesarias para incursionar en el mundo de los negocios, así como la capacidad para conocer las diferentes formas en las que el dinero se incrementa a través del tiempo, como los tipos de descuentos, anualidades, depreciaciones, capitalizaciones; entre otros. Ello, con el propósito de disponer recursos intelectuales para resolver problemas prácticos sobre las operaciones financieras que se presentan cotidianamente en las finanzas de todos los negocios, sean estos personales, empresariales, públicos o privados.

Como lo expresa (Shuell, 1986), aprender es un cambio perdurable de la conducta o en la capacidad de conducirse de manera dada, como resultado de la práctica o de otras formas de experiencia para adquirir nuevos conocimientos de otras materias. Enfatizamos en lo que resalta este autor, para vincular lo abordado anteriormente sobre el uso de las Matemáticas Financieras por parte de los estudiantes que la cursan a partir del tercer año de su carrera, quienes mejorar sus aprendizajes y fortalecen sus conocimientos, tan necesarios para poder entender otras materias claves para su desempeño profesional. 


\section{CONCLUSIONES}

La Matemática Financiera es una asignatura muy popularizada, que ha venido a enriquecer el currículo académico de las carreras que ofrece el Departamento de Ciencias Económicas y Administrativas, en la UNAN-Managua, FAREM-Estelí.

Es importante que todos los estudiantes de las carreras administrativas y contables, así como las económicas, mercadeo y finanzas, se apropien de sus contenidos, tan relevantes para crear mejores aprendizajes orientados al mundo de los mercados financieros, tomando en cuenta el valor del dinero en el tiempo, las tasas de interés, las rentas financieras, los créditos, fondos de amortización y análisis de las inversiones. En suma, es de mucha utilidad para la toma de decisiones orientadas en el campo económico, administrativo, financiero y contable de los negocios.

Es oportuno mencionar que el estudio de esta asignatura representa para los estudiantes un aspecto sustancial en la adquisición de mejores conocimientos, así como capacidades y habilidades al momento de llegar a ejercer su profesión, así como el estudiante se encontrará en el futuro en el mercado laboral con mejor competencia para enfrentar retos que se le presentarán.

\section{REFERENCIA BIBLIOGRÁFICA}

Delfin, L. (2008). Fundamentos de Matemática Financiera. México: Ecasa.

López, U. O. (2012). Matematica Financiera II. Ciudad de México .

Portus, L. (2005). Matemática Financiera. Bogotá: Mc Graw Hill Interamericana.

Reyes, N. (2004). Introduccion a la Gestión Financiera. Managua: Xerox, UCA.

Shuell, T. J. (1986). Introducción al aprendizaje.

UNAN-Managua (2011) Modelo Educativo de UNAN Managua. Managua. 